\title{
6
}

\section{A Repository System for Business Rules}

\author{
H. Herbst, T. Myrach \\ Institute of Information Systems \\ Research Unit 'Information Engineering' \\ University of Berne \\ Engehaldenstr. 8, CH-3012 Berne, Switzerland* \\ \{herbst|myrach\}@ie.iwi.unibe.ch \\ http://www.ie.iwi.unibe.ch
}

\begin{abstract}
:
In the last few years, rules as a new focus in systems analysis have been of increasing interest. One reason for this are recent developments in active database research which have resulted in first commercially available database management systems with active components. To support this rule-based active mechanisms, it is desirable that rules can be taken into account on the conceptual level. Another important reason for focusing on rules is their importance within organizations; in this context the term business rules is used. This paper defines and structures business rules as a main component of systems analysis, presents results of a case study, and introduces a meta-model and a repository system for business rules.
\end{abstract}

Keywords:

Business Rule, Repository, Dynamic modeling, Active Database, ECA

\section{INTRODUCTION}

Active databases are an important issue in current database research (cf. [StHH87]; [DaBM88]; [WiCL91]; [HaWi93]; [ChMi93]; [Gatz94]). The introduction of database triggers in commercial database management systems (DBMS) such as Ingres, Oracle, or Sybase are first approaches to make this research available for the development of information systems (IS). According to this progress on the implementation level, the importance of treating business rules on the conceptual level is evident (cf. [App184]; [VLLS88]; [BBSG90]; [Mori93]; [KnHe93]). Though the term business rule is often referenced, it is defined and used rather differently and often restricted to semantic integrity constraints (cf. e.g. [Appl88]; [SaVo91]).

\footnotetext{
* The work presented in this paper has been partially supported by the Swiss National Science Foundation, Priority Programme Informatics, Project 5003-034330.
} 
However, business rules do not only cover data integrity but may also impose restrictions on organizational dynamics; therefore, we define business rules with reference to [BBSG90] as statements about how the business is done, i.e., about guidelines and restrictions with respect to states and processes in an organization [Herb95].

A comparison of selected function-oriented, data-oriented, and object-oriented methods revealed that no single method allows a convenient graphical specification of different aspects of business rules on the conceptual level [HKMS94]; however, a combination of several methods, each representing a specific view on business rules, may be applied. To complement ongoing research dealing primarily with the graphical representation of business rules (cf. e.g. [Tana92], [MaOd92]), we focus on the administration of business rules in a repository system from which different (e.g. graphical) views can be generated.

\section{BUSINESS RULES}

\subsection{Systems Analysis Focusing on Business Rules}

In systems analysis a main goal is to collect all relevant information about the universe of discourse on a conceptual level [Poh193]; these facts are primarily about processes and the structure and manipulation of data objects. Business rules as defined above encompass these two aspects; therefore, we regard business rules as a central element of systems analysis and define the procedure of systems analysis accordingly (cf. Figure 1). The following two steps are the current focus of the implementation of the repository system described in this paper:

1. Step: Facts about the universe of discourse are collected in the real world (e.g. by interviews or document analysis) and specified as business rules.

2. Step: A major part of relevant entity types, relationship types, and attributes are derived from the collection of business rules and afterwards structured in a conceptual data model.

The subsequent task of checking the integrity between the data model and the business rule collection may lead to an iteration. Finally, the specifications can be used in systems design and the implementation, e.g., in an active database. 


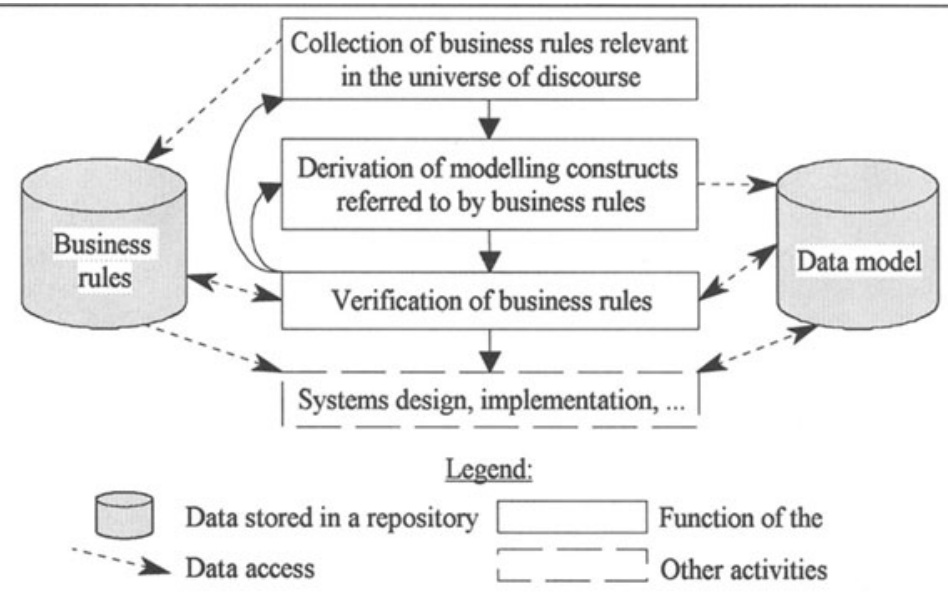

Figure 1: Systems analysis focusing on business rules

\subsection{The Structure of Business Rules}

Rules in active databases (cf. [DaBM88]) are structured according to the Event-ConditionAction (ECA) paradigm (cf. [TsKL90]; [HaWi93]). Because one of our goals is to derive implementations of business rules in active components, it seems to be appropriate to structure business rules accordingly. As a result of several case studies to extract business rules from practically applied IS [KnHS94], we use ECAA rules which can always be transformed into one or two ECA rules by negating the condition. The semantic of the components is as follows:

- Event: When has a business rule to be processed?

- Condition: What has to be checked?

- Then-Action: What has to be done if the condition is true?

- Else-Action: What has to be done if the condition is false?

These basic component types of business rules can be further classified into several subtypes [HeKn94] which is especially relevant for the definition of a syntax for specifying business rules in a repository system.

The ECAA structure allows to specify integrity constraints as well as entire processes consisting of business rules as basic elements. The possibility to specify processes using ECAA rules makes business rules applicable within the concepts of business process re-engineering [HaCh93]. The importance of events with respect to processes is emphasized in [Stre94] and there exist several event-oriented process description approaches (cf. e.g. [ÖsGu92], [Sche94]); in the research of active databases, several event algebras have been proposed (cf. e.g. [GeJS92]; [Gatz94]; [CKAK94]). Even though the dividing line between an integrity constraint and a process step is fuzzy, some approaches try to separate these two constructs. This 
artificial separation may lead to a gap in the process specification because the evaluation of a semantic integrity constraint may be part of a process and its result could trigger process steps. In our approach, an integrity condition corresponds to the condition part of a business rule which encompasses additionally the event which triggers the evaluation of the constraint predicate and a (re)action depending on the result.

For the specification of a process it is necessary to link the action-component of a business rule to events which may trigger other business rules. This link is explicitly specified within the action-component by indicating which events are raised. For reasons of having a clear structure, we do not allow to raise two exclusive events within a single action; such a situation has to be circumvented by replacing the business rule by two or more rules.

\subsection{Case Studies on Business Rules}

\subsubsection{Overview and Examples}

In our research project, three case studies have been carried out (cf. [KnHS94], [KnSc94]) to extract business rules from practically applied IS. The aim of these case studies was to get insights in different types of rules and their relevance. Figure 2 gives an overview on the studies.

The business rules discussed in this paper are extracted from the case study dealing with the insurance IS „DOM-2“ which automates the processing of insured events, i.e., damages. This IS covers personal liability insurances, third party insurances, insurances against theft, and other property insurances. In the case study two different aspects have been taken into account: (1) the organizational process of treating insured events and (2) the IS needed within the process. The organizational process has been analyzed by interviewing the staff of the involved department. These interviews revealed 150 rules controlling the process of the damage administration; the analysis of the COBOL source code resulted in approximately 600 additional rules like the following:

ON (damage-field entered) OR (damage-cause entered)

IF $\quad$ (damage-field = private third party insurance) $A N D$

(damage-cause = damage of a car in use) $A N D$

(third-party-insurance-type = family, single or senior)

THEN issue error message „Damages of cars in use are not insured by this policy; please check and if necessary, pass the file to the central office. “

This example illustrates the difficulties of separating integrity constraints and process steps because the negative evaluation of the condition may lead to passing the case to the central office; this is certainly relevant for the business process and must not be excluded from its specification. 


\begin{tabular}{|c|c|c|c|}
\hline & ${ }_{n} \mathrm{DAZ}$ & ${ }_{n} \mathrm{DOM}-2^{4}$ & ${ }_{n} \mathrm{BALICO}^{4}$ \\
\hline Enterprise & Swiss bank company & Swiss insurance company & Swiss PTT \\
\hline Purpose of the IS & $\begin{array}{l}\text { Decentral entry of pay- } \\
\text { ments }\end{array}$ & $\begin{array}{l}\text { Entry and administration } \\
\text { of damage claims }\end{array}$ & $\begin{array}{l}\text { Administration of rented } \\
\text { and owned buildings }\end{array}$ \\
\hline $\begin{array}{l}\text { Development } \\
\text { environment }\end{array}$ & $\begin{array}{l}\text { COBOL, } \\
\text { IMS }\end{array}$ & $\begin{array}{l}\text { COBOL, } \\
\text { IMS }\end{array}$ & $\begin{array}{l}\text { Oracle*Forms } 3, \\
\text { Oracle V6 }\end{array}$ \\
\hline Research Methods & Code analysis, interviews & Code analysis, interviews & Code analysis \\
\hline Lines of code (approx.) & $1 ' 500$ & $80 ’ 000$ & $55^{\prime} 000$ \\
\hline Number of business rules & $76 \quad(100 \%)$ & $758 \quad(100 \%)$ & $523 \quad(100 \%)$ \\
\hline ... in application code & $(84 \%)$ & $(83 \%)$ & $523 \quad(100 \%)$ \\
\hline ... in environment & $12 \quad(16 \%)$ & $(17 \%)$ & $(0 \%)$ \\
\hline
\end{tabular}

Figure 2: Overview on the case studies

To illustrate the scope and different types of business rules, we introduce a set of rules from the case study describing a part of the process on a general level. The process is initiated by any person getting in contact with the insurance company. The first business rule encompasses checks whether the person is a policy-holder of our company and whether the policy-holder wants to report a damage.

Business rule 'PERSON-CONTACTS-US':

ON (PHONE-CALL-OF-PERSON) OR (LETTER-OF-PERSON)

IF (person is a policy-holder) AND (policy-holder wants to report a damage)

THEN begin damage registration raise event 'DAMAGE-REPORTED'

The processing of this business rule leads to the provisional registration of the damage if the policy-holder does already provide us with information on the damage; in the other case he receives a form to fill in.

Business rule 'REGISTER-PROV-DAMAGE':

ON damage reported

IF (information about damage available)

THEN register damage provisionally

registration-date $:=T O D A Y O$

raise event 'DAMAGE-PROV-REGISTERED'

ELSE send damage form to policy-holder

raise event 'DAMAGE-FORM-SENT'

The registration of the incomplete information about the damage is followed by a check whether the damage is covered by a policy of the policy-holder. Depending on the result, either the claims of the policy-holder are rejected or a form to complete the information about the damage is sent to the policy-holder. 
Business rule 'ACCEPT-PROV-DAMAGE':

ON provisional damage registered

IF (damage insured by a policy)

THEN accept damage provisionally send damage form to policy-holder raise event 'DAMAGE-FORM-SENT' raise event 'DAMAGE-PROV-ACCEPTED'

ELSE reject damage raise event 'DAMAGE-REJECTED'

The policy-holder is reminded if he does not return the form within 60 days:

Business rule 'REMINDER-FOR-DAMAGE-FORM':

ON 60 days after (DAMAGE-FORM-SENT)

IF (damage form not returned)

THEN remind policy-holder

Another 30 days later, the provisionally registered damage is deleted if the form has not been returned.

Business rule 'TIME-OUT-FOR-DAMAGE-FORM':

ON 90 days after (DAMAGE-FORM-SENT)

IF (damage form not returned)

THEN delete provisionally accepted damage

The last rule in the example is triggered by the two events 'damage form sent' and 'damage form received' which have to occur in conjunction. Every received form is checked with regard to its completeness and returned to the policy-holder if it is incomplete. Otherwise the definitive registration of the damage can be started.

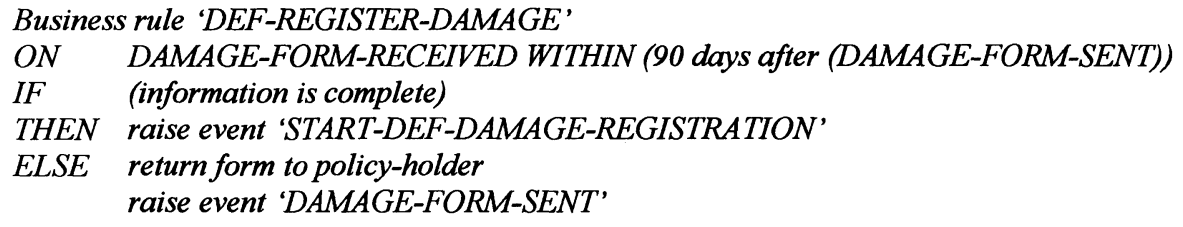

Subsequent to the execution of these business rules, the process of treating the definitive damage claims of the policy-holder may start.

\subsubsection{Requirements for Active Components in DBMS resulting from the Case Studies}

The business rules extracted in the case studies give an overview of practically relevant rules and therefore allow to draw some requirements for active components in DBMS. This research is complemented by a technical evaluation of database triggers and a survey of users. The 
evaluation considered three commercially available DBMS with active components (i.e. database triggers): CA-Ingres V6.4, Oracle V7.0, and Sybase V10. The following main differences between these three DBMS have been found [KnHS94]:

- In Sybase triggers are processed for each statement, in CA-Ingres for each referenced tuple and in Oracle alternatively for both.

- Ingres allows an infinite number of database triggers raised by a single event; therefore, business rules may be implemented modularly. In Oracle and Sybase the number of triggers per event is restricted ( 12 or 3 respectively).

- Oracle and Sybase do only support events raised by a data manipulation (Insert, Update and Delete); CA-Ingres allows to specify a kind of user defined events.

- In Oracle a trigger can not manipulate data in the table related to the triggering event because this table is locked by the executed database trigger.

Furthermore, a survey of CA-Ingres, Oracle, and Sybase users has been conducted with respect to the practical application of database triggers [Rytz94]. The main problems reported from the users were the missing support of rules on the conceptual level, the missing standardization of database triggers, experienced (or feared) performance problems, and the insufficient tools for the administration of implemented database triggers.

From those research activities, some problems of implementing the business rules found in the case studies can be derived:

- In the case study „DOM-2“, the maximum number of business rules triggered by a single event (modification of the damage cause) was approximately 230. The implementation of these rules using e.g. Oracle or Sybase leads to a very large non-modular database trigger.

- It is obvious that the high number of rules triggered by single events can easily lead to performance problems.

- Time related events as e.g. the ones presented in the example can not be implemented because none of the DBMS regarded supports the specification of this type of events.

The implementation of business rules as those found in the case study demand the following functionalities of an active DBMS:

- To provide modularity, the number of rules per event must not be restricted.

- An active DBMS should provide a various number of event types as listed in [ChMi93].

- To support the administration of rules implemented in an active DBMS, several tools have to be developed.

Furthermore, as mentioned above, rules have to be considered on the conceptual level to provide a basis for their implementation. For this purpose, the meta-model and the repository system presented in the following chapters have been developed. 


\section{A BUSINESS RULE ORIENTED META-MODEL}

\subsection{The Core Submodel 'Business Rule'}

The submodel 'business rule' consists of the four meta-entity-types Business rule, Event, Condition, and Action (cf. Figure 3). According to the structure presented in the introduction, a business rule encompasses exactly one event, at most one condition, and one or two action components (then/else). Furthermore, business rules can be specialized and generalized which leads to a recursive m:n relationship on Business rule. The meta-model allows the specification of complex events as, e.g., time-related events and the conjunction or disjunction of events. Complex events always make reference to at least one other event which is expressed by a m:n relationship on Event. A similar recursive relationship exists for the meta-entity-type Condition. As discussed in the examples, actions of business rules may raise events which is depicted in the meta-model as a relationship between the meta-entity-types Action and Event.

\subsection{Embedding Business Rules into their Environment}

Business rules as defined and exemplified above describe how the business is done; however, to obtain an integrated view on the universe of discourse, they have to be embedded into their environment. In Figure 3, an overview on the relationships between business rules and the following submodels is given:

- Software components: Components of business rules may be implemented in a software component as, e.g., a module, a database trigger or a stored procedure.

- Organizational unit: Business rules can be allocated to organizational units from either the viewpoint which organizational units are responsible for the content of a business rule or which organizational units have to process the different components of a business rule.

- Process: Business rules structured as ECAA rules are adequate to specify processes. The concept of specialization discussed above allows to define different process levels where each (sub)process encompasses a finite set of business rules. Processes are furthermore closely linked to organizational units.

- Origin: Each of the real world facts specified as business rules has a specific origin as, e.g., an organizational guideline. The relationship between business rules and their origin supports the administration and especially the validation of the business rule collection.

- Modeling construct. The submodel 'modeling construct' encompasses the meta-entitytypes for the specification of a data model. Because of its widely accepted standards, we chose the Entity Relationship model, but the meta-model could easily be adapted to another data model as, e.g., an object-oriented model, because all the rule components only relate to the generalized meta-entity-type modeling construct. The representation of the different views on the relationship between modeling constructs and business rules is discussed within the subsection 4.3 . 
By specifying the relationships between business rules and other submodels, business rules can be classified e.g. according to the approach discussed in [Herb95] and [HeKn94] which supports the administration of large rule sets.

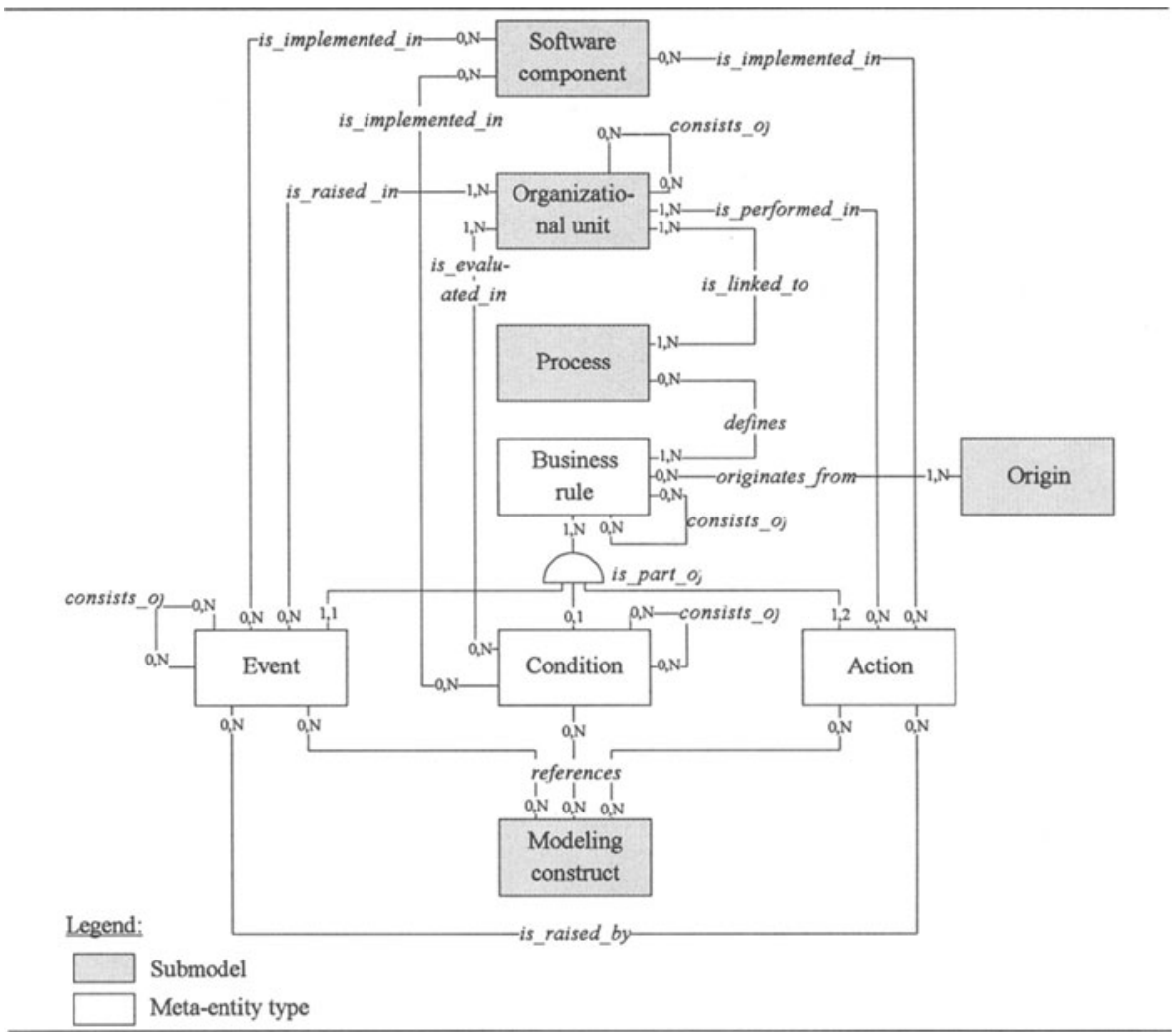

Figure 3: Business rules embedded into their environment

\section{A REPOSITORY SYSTEM FOR BUSINESS RULES}

\subsection{Functionality of the Repository System}

A full-fledged repository system is on principle a DBMS with some special functionality to administer meta-data (cf. [Myra95]). This includes the capability to specify meta-models, to customize the user interface, and to implement appropriate verification mechanisms and generators. To cope with the large number of meta-data, a repository system has to provide for 
sophisticated query possibilities; these should support semi-graphical and/or graphical representations of query results.

Instead of building a repository-system from scratch, we decided to base it on an available solution which can be adapted to our specific purpose. Applied to the administration of business rules, the employed repository system must support a dictionary-schema that is equivalent to the meta-model introduced in chapter 3. Because this meta-model is particular to our approach, the system has to allow for user-defined meta-schemas. The system has to be adapted to support the tasks (1) business rule registration, (2) verification of the business rules (e.g. checking for loops), (3) derivation of modeling constructs, and (4) specification of the data structure. To this end, a flexible user interface and a powerful programming language have been needed. Furthermore, we demanded a graphical component to support different notations for the representation of business rules, e.g., those discussed in [HKMS94]. A commercial product that largely satisfies these requirements and that we have therefore chosen for our implementation is the repository system Rochade.

\subsection{Implementation of the Meta-Model and a User-Interface}

In Rochade, the basic constructs to describe meta-models are documents, chapters and links; these constructs constitute the dictionary schema definition layer according to the 4-levelarchitecture of the IRDS standard [ANSI89]; the implementation of the meta-model 'business rule' and the related submodels by means of these constructs then constitutes the dictionary definition layer in this terminology. On this layer, the meta-entity-types have to be implemented as documents of diffent types (e.g. of the type BUSINESS RULE or EVENT). The relationship-types of the meta-model have to be implemented as directed links between a source and a target document. The building of a link to a document is triggered by the occurence of a defined keyword in a linking chapter of the source document. Thus, e.g., the keyword $\rightarrow E V E$ in a document of the type BUSINESS RULE results in a link to a document of the type EVENT.

Following the definition of the dictionary schema, a user interface for the manipulation of business rules has been developed. This user interface is based on three main windows. The first window is devised for a fast registration of business rules only. The references within the business rule documents result in links to other documents which might not (yet) exist; a manipulation of the referenced documents is not possible within the framework of this window. The second window serves for the manipulation of business rules in their context; it allows as well the manipulation of entity types, relationship types, and attributes. Furthermore, it supports the analysis of the relationships between registered business rules and modeling constructs. The third window deals with the (static) process structure which is used for the specialization of business rules.

Figure 4 depicts the second window of the user interface including our example rules; it is overlaid by a sub-window for the registration and manipulation of a single business rule. The example shows how the relationships between the business rule PERSON-CONTACTS-US and instances of other meta-entity-types have to be specified within the rule syntax (e.g. $\rightarrow E N T$ $D A M A G E$ as a reference to the document DAMAGE of the type ENTITY TYPE and $\rightarrow E V E$ $D A M A G E-R E P O R T E D$ as a reference to the event raised by the action). 


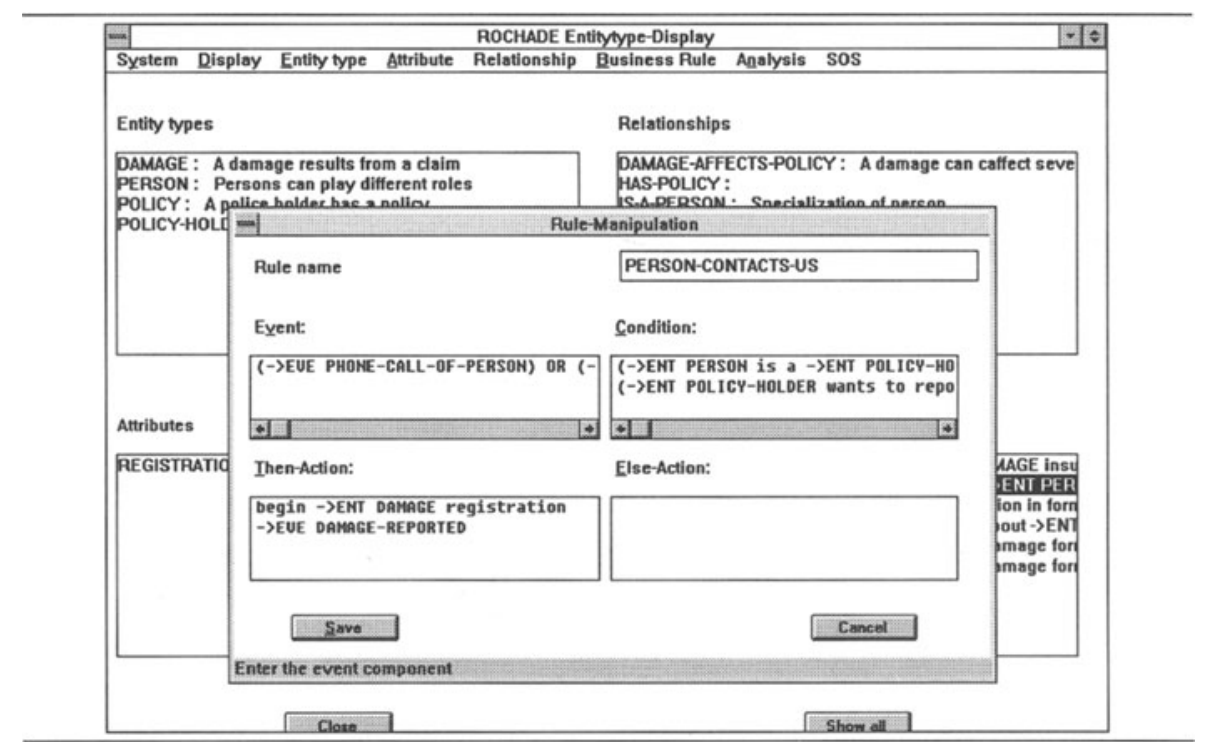

Figure 4: Window for manipulating a single business rule

The case studies revealed the amount of business rules in a real system as a major problem. This results in the need for mechanisms to reduce complexity; therefore, a concept of generalization and specialization of business rules has been devised and implemented in the repository system. This approach adapts the principles of leveled dataflow diagrams (DFD) as used in Structured Analysis [DeMa78]; in leveled DFD, the parent and the child diagram have to be balanced, i.e., "data flows into and out of the parent bubble are equivalent to data flows into and out of the child diagram". A straightforward adaptation of this balancing-rule to the specialization of business rules results in the condition that on both levels the triggering events and the raised events have to be identical (for an example cf. [Herb95]).

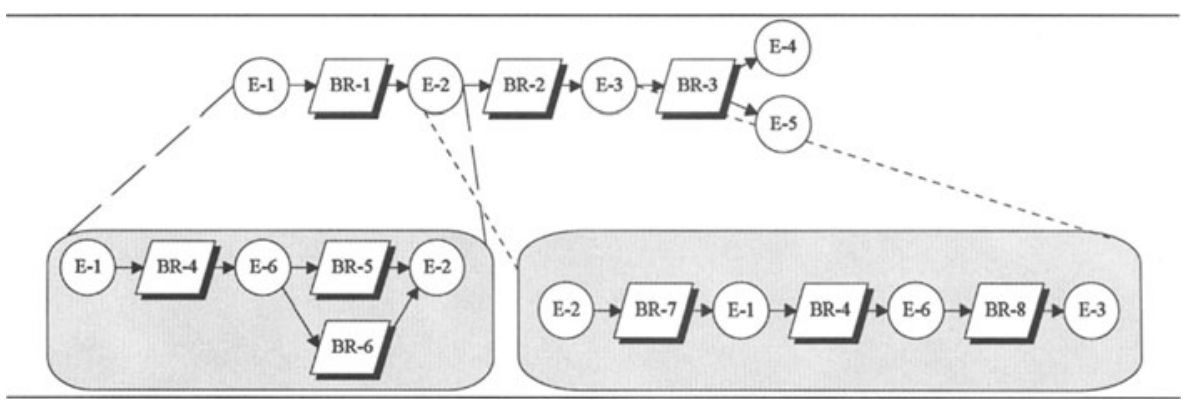

Figure 5: Specialization of business rules 
In our concept, different levels for specifying business rules are allocated to (sub)processes encompassing a specific subset of business rules (cf. Figure 5). This allocation allows a reuse of business rules on different levels (e.g. the rule 'BR-4' in Figure 5). The reused business rules always raise the same events but may trigger different business rules, depending on the subset of business rules which are element of the current subprocess. As mentioned above, the process structure is manipulated in a particular window which is depicted in Figure 6.

\begin{tabular}{|l|}
\hline System Current process Sub process Analysis SOS \\
\hline Super processes \\
DOM-2: Main process of the case study \\
Current process - \\
DAMAGE-REGISTRATION \\
Registration of a damage claim \\
Sub processes \\
REGISTER-PAGE-0: Enter data of page 0 \\
REGISTER-PAGE-1: Enter data of page 1 \\
REGISTER-PAGE-2: Enter data of page 2 \\
REGISTER-PAGE-3: Enter data of page 3 \\
\hline
\end{tabular}

Figure 6: Window for the manipulation of the process structure

\subsection{Graphical Representation of Business Rules documented in the Repository System}

An important element of the repository system for business rules is the capability to represent graphically the contents of a repository. For visualizing different views on business rules, we have chosen the following models:

- Model of the static process structure

- Entity-Relationship model (ERM)

- $\mathrm{ER}^{2}$ model or the earlier Entity-Relationship-Rule model respectively [TNCK91]

- Event-Rule (E/R) network as a high-level Petri Net

These models have been implemented using Rochade-Graphics. The resulting graphical representations are exemplified in the following by the business rules introduced in chapter 2.3.1. The diagrams depicted are entirely generated by the repository system; only the layout of the diagrams has been manually adapted.

\subsubsection{Process Structure}

The diagram for representing the process structure includes a hierarchical network of superand subprocesses. In these diagrams different types of rectangles are used to represent whether a subprocess is already (at least partly) specified by business rules or not. Likewise, different 
types of arrows symbolize whether a subprocess has been connected to a specialized business rules on the higher process level or not.

To reduce the size and complexity of the $\mathrm{ER}^{2}$ models and the ER networks presented in the following subsections, the diagrams are drawn for each of the specialized subprocesses. The example business rules introduced in this paper describe the subprocess 'Damage-Reception'; therefore, the following diagrams are generated for this subprocess.

\subsubsection{Entity-Relationship Model}

As discussed in the description of a business rule based systems analysis (cf. Figure 1), modeling constructs can be derived from a collection of business rules and afterwards structured in a data model. The modeling constructs referenced in the examples and represented in Figure 7 using the ERM are:

- Entity types 'Person', 'Policy-Holder', 'Damage', and 'Policy'

- Attribute 'Registration-Date'

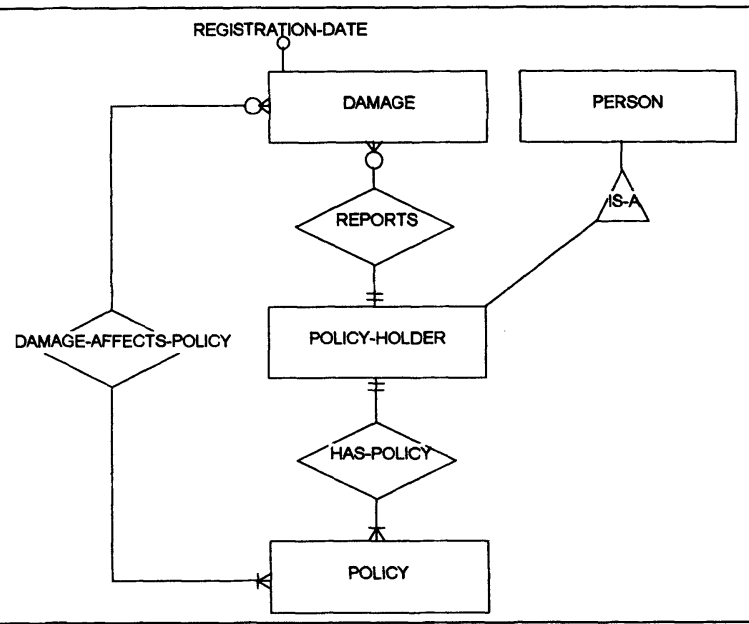

Figure 7: ERM resulting from the business rules of the example

This ERM visualizes that a policy-holder must have at least one policy. He may report damages which affect at least the policy of the policy-holder but can also affect policies of other policy-holders, e.g. if several cars involved in a particular car crash are insured by policies of the company regarded. 


\subsection{3 $\mathrm{ER}^{2}$ : Entity-Relationship-Event-Rules Model}

The submodels 'business rule' and 'modeling construct' allow a view on the meta model focusing on the relationship between modeling constructs and business rules; it can be regarded from the following points of view:

1. Business rule $\Rightarrow$ modeling construct: Which modeling constructs are used by a specific business rule and which impact does it have on modeling constructs?

$\begin{array}{ll}\text { Example: The business rule 'Person-Contacts-Us' makes reference to } \\ \text { - entity type 'Policy-Holder' } \\ \text { - entity type 'Person' } & \text { (condition) } \\ \text { - entity type 'Damage' } & \text { (condition) }\end{array}$

2. Modeling construct $\Rightarrow$ business rule: Which business rules use a specific modeling construct?

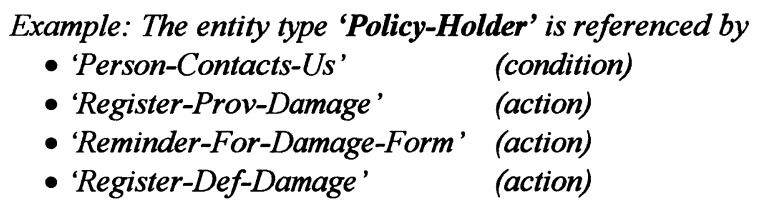

As discussed above, these views on the relationship between business rules and modeling constructs may be depicted in an $\mathrm{ER}^{2}$ model [Tana92]. This model suits especially the visualization of business rules because it has been developed for depicting ECA rules of active databases on the conceptual level. An $E^{2}$ model encompasses an ERM and combines it with a simple Event-Rule net. In this net, events are drawn as circles and the condition-action part of a business rule is depicted as a parallelogram. Events may trigger condition-action constructs and complex events; this triggering is depicted by an arrow. In the original $\mathrm{ER}^{2}$ model, all relationships between the constructs of the ERM and the net are drawn; to reduce the complexity resulting from the large amount of connections, we decided to use different layers for the event, condition, and action component of a rule; these layers may also be depicted simultaneously. Figure 8 shows the impact of the business rules on the modeling constructs, i.e., the relationships between the action components of the business rules and the modeling constructs. Dotted lines are used to separate these relationships from the arcs between events and rules. The events symbolized by a dashed line are referenced but not stored in the repository yet; they exist only virtually. The diagram is further enriched by indicating the complex event types. 


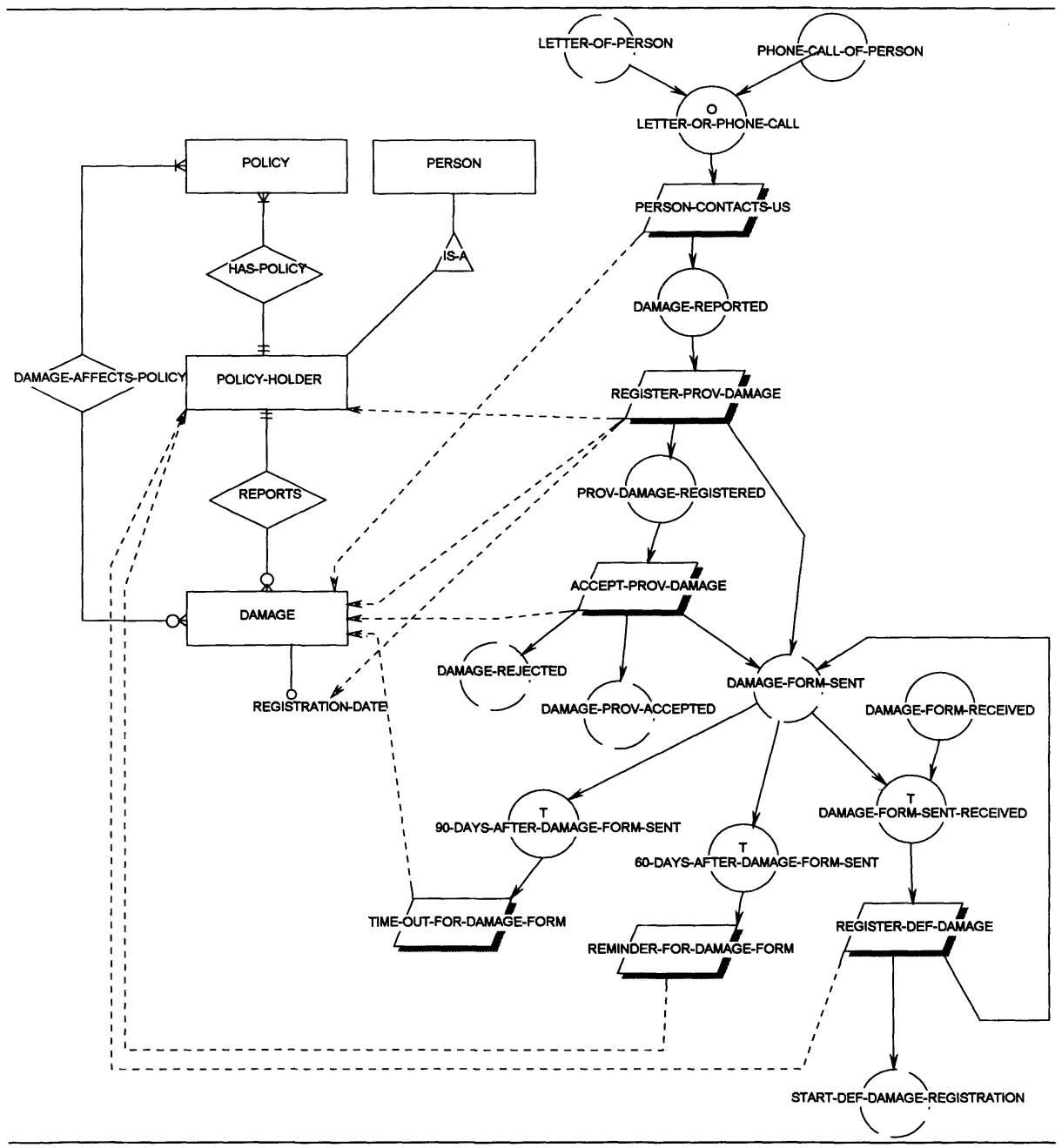

Figure 8: $\mathrm{ER}^{2}$ model with references from rule actions to the ERM 


\subsubsection{High-level Petri-Net}

The dynamics of business rules may be represented by Petri Nets [HKMS94]. In [Tana92] the Event-Rule (E/R) network is derived from the $E^{2}$ diagram. An E/R network is a high-level Petri Net defined by the triple $(E, R, A): E$ is a finite set of places representing events, $R$ is a finite set of transitions representing rules (condition/action part of a business rule) and $A$ is a finite set of directed arcs representing the raising of events and the triggering of rules.

To match the requirements of a Petri Net, the rather simple representation of the dynamics in the $\mathrm{ER}^{2}$ diagram has to be modified. In [Tana92] the transformation of two cases is discussed:

(1) The triggering of a rule by several disjunctive events and (2) the concurrent triggering of several rules by a single event. Because we extended the ECA structure by a THEN/ELSE structure, another transformation (3) has to be considered which handles mutually exclusive events raised by a business rule (cf. Figure 9).

(1)

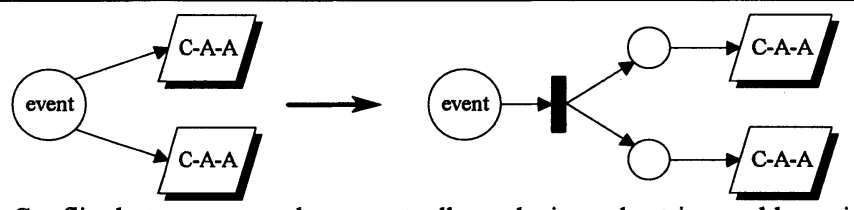

Conflict between several non-mutually exclusive rules triggered by a single event

(2)
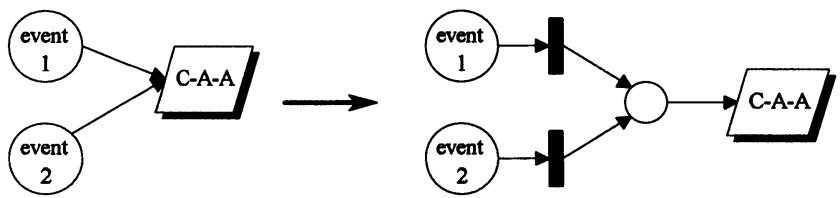

Coordination between several disjunctive events triggering a single rule

(3)

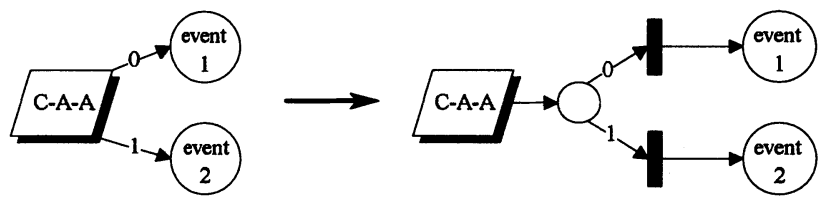

Conflict between mutually exclusive events as result of the THEN-ELSE-structure

Figure 9: Rules for deriving an $\mathrm{E} / \mathrm{R}$ network from the $\mathrm{ER}^{2}$ diagram

The application of these transformations on the $\mathrm{ER}^{2}$ diagram of Figure 8 leads to the E/R network depicted in Figure 10. This diagram also exemplifies that loops may appear in organizational processes; therefore, they must not be forbidden within such a net. To support the administration, those loops may be indicated in the diagram, but the decision whether to remove them has to be left to the user. 


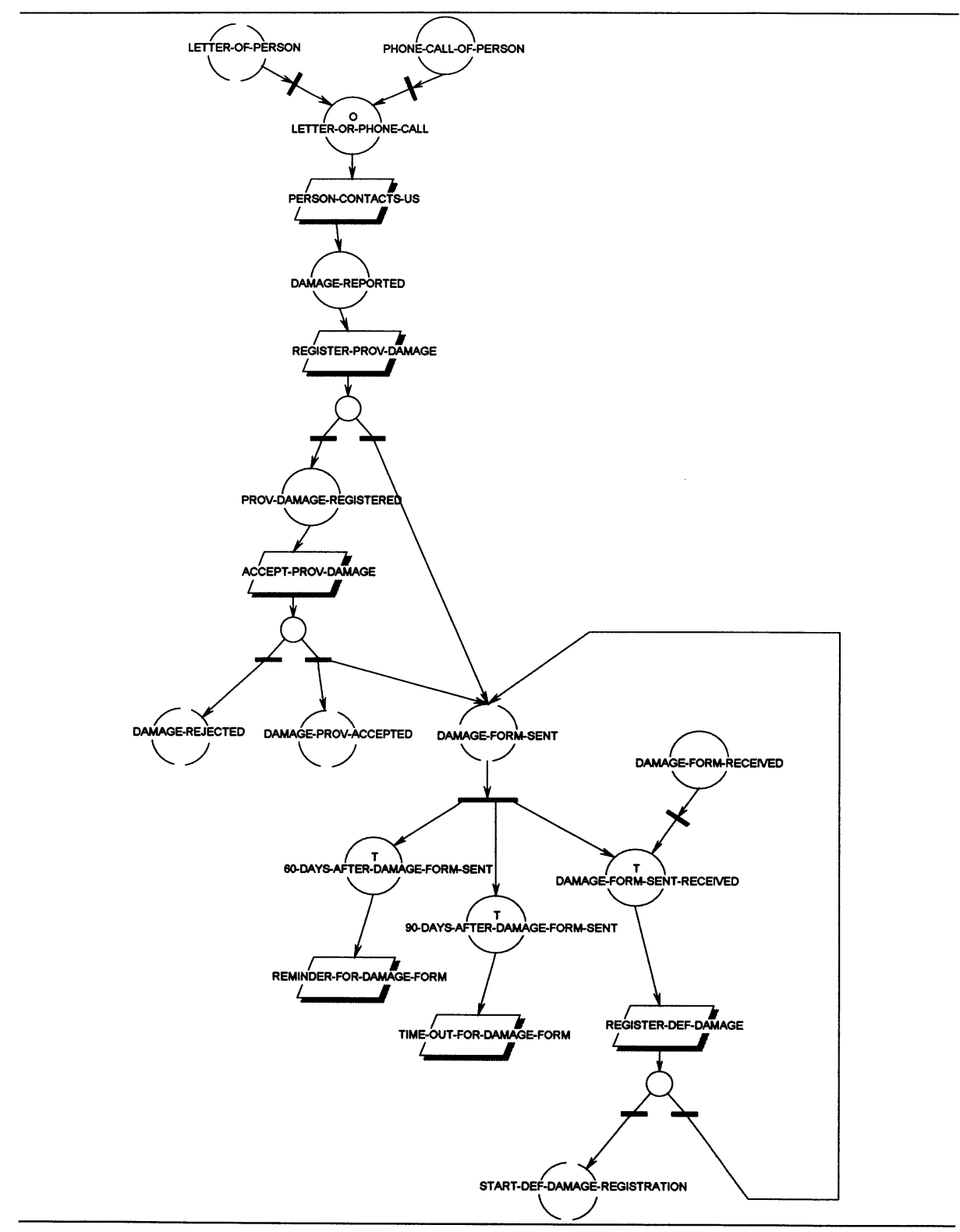

Figure 10: E/R network for the business rules of the example 


\section{CONCLUSIONS AND OUTLOOK}

According to research in the area of active databases and to proposals for graphical representations of rules implemented in those databases, a meta-model for the treatment of business rules on the conceptual level has been developed. In this meta-model, business rules are related to data modeling constructs and embedded into their organizational environment. This embedding gives various possibilities for the analysis and administration of business rules. Business rules as a technique for systems analysis fulfill requirements of systems users and analysts as, e.g., the ones described in [McGi92].

A commercial repository system has been used to implement the meta-model, a user-interface, and different graphical representation techniques for business rules. This business rule repository system was employed for the documentation of a large case study dealing with an insurance application. To support the administration of such a large rule-base, a concept for specialization and generalization of business rules has been incorporated. The implemented graphical models provide a visualization of views on business rules and help to communicate them, e.g., to involved end-users. Furthermore, the property to implement user-defined graphical representations may be employed for testing the applicability of different event-oriented approaches (cf. e.g. [RoFB88]; [VLLS88]; [MaOd92]; [Sche94]) on a realistic case study.

Finally, to close the gap between the conceptual and physical level, the repository system should be complemented by functions for the determination and derivation of implementation alternatives such as stored procedures or database triggers. Furthermore, the development and implementation of algorithms for checking the consistency of business rules and for providing consistency between business rules and the data model is desirable. Another research issue is integrating our business rule concept in workflow tools for business process re-engineering.

\section{REFERENCES}

[ANSI89] ANSI, American National Standard X3.138-1988: Information Resource Dictionary System (IRDS), New York: American National Standard Institute 1989.

[Appl84] Appleton, D.S., Business Rules: The Missing Link, in: Datamation 30 (1984) 16, pp. 145 - 150.

[Appl88] Appleton, D.S., Second Generation Applications, in: Database Programming \& Design 1 (1988) 2, pp. 48 - 54.

[BBSG90] Bell, J., Brooks, D., Goldbloom, E., Sarro, R., Wood, J., Re-Engineering Case Study Analysis of Business Rules and Recommendations for Treatment of Rules in a Relational Database Environment, Bellevue Golden: US West Information Technologies Group 1990.

[CKAK94] Chakravarthy, S., Krishnaprasad, V., Anwar, E., Kim, S.-K., Composite Events for Active Databases: Semantic Context and Detection, in: Proceedings of the VLDB 1994, pp. 606 - 617.

[ChMi93] Chakravarthy, S., Mishra, D., Snoop: An Expressive Event Specification Language for Active Databases, Technical Report UF-CIS-TR-93-007, University of Florida, 1993.

[DaBM88] Dayal, U., Buchmann, A.P., McCarthy, D.R., Rules Are Objects Too: A Knowledge Model for an Active, Object-Oriented Database Management System, in: K.R. Dittrich (Eds.), Advances in Object-Oriented Database Systems, Berlin et al.: Springer 1988, pp. 129 - 143.

[DeMa78] De Marco, T., Structured Analysis and System Specification, New York: Yourdon 1978.

[Gatz94] Gatziu, S., Events in an Active Object-Oriented Database System, Hamburg: Kovac 1994.

[GeJS92] Gehani, N.H., Jagadish, H.V., Shmueli, O., Event Specification in an Active Object-Oriented Database, in: $A C M$ SIGMOD 21 (1992) 2, pp. 81 - 90.

[HaCh93] Hammer, M., Champy, J., Reengineering the corporation, New York: Harper Business 1993. 
[HaWi93] Hanson, E.N., Widom, J., An Overview of Production Rules in Database Systems, in: Knowledge Engineering Review 8 (1993) 2, pp. 121 - 143.

[Heil94] Heilmann, H., Workflow Management: Integration von Organisation und Informationsverarbeitung, in: Handbuch der modernen Datenverarbeitung (1994) 176, pp. 8 - 21.

[Herb95] Herbst, H., A Meta-Model for Specifying Business Rules in Systems Analysis, in: J. Iivari, K. Lyytinen, M. Rossi (Eds.), Proceedings of the Seventh Conference on Advanced Information Systems Engineering, Berlin et al.: Springer 1995, pp. 186 - 199.

[HeKn94] Herbst, H., Knolmayer, G., Ansätze zur Klassifikation von Geschäftsregeln, in: Wirtschaftsinformatik 37 (1995) 2, pp. 149 - 159.

[HKMS94] Herbst, H., Knolmayer, G., Myrach, T., Schlesinger, M., The Specification of Business Rules: A Comparision of Selected Methodologies, in: A.A. Verrijn-Stuart, T. W. Olle (Eds.), Methods and Associated Tools for the Information System Life Cycle, Amsterdam et al.: Elsevier 1994, pp. $29-46$.

[KnHe93] Knolmayer, G., Herbst, H., Business Rules, in: Wirtschaftsinformatik 35 (1993) 4, pp. 386 - 390.

[KnHS94] Knolmayer, G., Herbst, H., Schlesinger, M., Enforcing Business Rules by the Application of Trigger Concepts, in: Swiss National Science Foundation (Ed.), Proceedings Priority Programme Informatics Research, Information Conference Module 1, Berne 1994.

[KnSc94] Knolmayer, G., Schlesinger, M., Die Transformierbarkeit von Applkationstriggern in Datenbanktrigger am Beispiel der Oracle-Entwicklungsumgebung und des BALICO-Systems, Working Paper 49, Institute of Information Systems, University of Berne 1994.

[MaOd92] Martin, J., Odell, J., Object-Oriented Analysis \& Design, Englewood Cliffs: Prentice-Hall 1992.

[McGi92] McGinnes, S., How Objective is Object-Oriented Analysis?, in: P. Loucopoulos (Ed.), Proceedings of the Forth International Conference on Advanced Information Systems Engineering, Berlin et al.: Springer 1992, pp. 1 - 16.

[Mori93] Moriarty, T., The Next Paradigm, in: Database Programming \& Design 6 (1993) 2, pp. 66 - 69.

[Myra95] Myrach, T., Konzeption und Stand des Einsatzes von Data Dictionaries, Heidelberg: Physica 1995.

[ÖsGu92] Österle, H., Gutzwiller, T., Konzepte angewandter Analyse- und Design-Methoden, Band 1: Ein Referenz-Metamodell für die Analyse und das System-Design, Hallbergmoos: AIT 1992.

[Pohl93] Pohl, K., The Three Dimensions of Requirements Engineering, in: C. Rolland, F. Bodart, C. Cauvet (Eds.), Proceedings of the Fifth International Conference on Advanced Information Systems Engineering, Berlin et al.: Springer 1993,pp. 275 - 292.

[RoFB88] Rolland, C., Foucaut, O., Benci, G., Conception des systèmes d'information - La méthode REMORA, Paris: Eyrolles 1988.

[Rytz94] Rytz, M., Stand der Nutzung von Datenbank-Triggern in der Anwendungsentwicklung - Eine empirische Untersuchung zum Einsatz von Triggerkonzepten in Oracle, Sybase und Ingres, Master Thesis, Institute of Information Systems, University of Berne 1994.

[SaV091] Sandifer, A., Von Halle, B., Linking Rules to Models, in: Database Programming \& Design 4 (1991) 7, pp. 13 - 16.

[Sche94] Scheer, A.-W., Wirtschaftsinformatik - Referenzmodelle für industrielle Geschaftsprozesse 5th ed., Berlin et al.: Springer 1994.

[StHH87] Stonebraker, M., Hanson, E., Hong, C.-H., The Design of the POSTGRES Rules System, in: Proceedings of the IEEE International Conference on Data Engineering 1987, pp. 365 - 374.

[Stre94] Streng, R.J., BPR needs BIR and BTR: The PIT-framework for Business Re-engineering, in: Proceedings of the Second SISnet Conference, Barcelona 1994.

[Tana92] Tanaka, A.K., On Conceptual Design of Active Databases, PhD Thesis, Georgia Institute of Technology 1992.

[TNCK91] Tanaka, A.K., Navathe, S.B., Chakravarthy, S., Karlapalem, K., ER-R: An Enhanced ER Model with Situation-Action Rules to Capture Application Semantics, in: T.J. Teorey (Ed.), Proceedings of the 10th International Conference on the Entity Relationship Approach, San Mateo: E/R Institute 1991, pp. 59 - 75.

[TsKL90] Tsalgatidou, A., Karakostas, V., Loucopoulos, P., Rule-Based Requirements Specification and Validation, in: B. Steinholtz, A. Sølvberg, L. Bergman (Eds.), Proceedings of the Second Nordic Conference on Advanced Information Systems Engineering, Berlin et al.: Springer 1990, pp. 251 - 263. 
[VLLS88] Van Assche, F., Layzell, P., Loucopoulos, P., Speltincx, G., Information systems development: a rule-base approach, in: Journal of Knowledge Based Systems 1 (1988) 4, pp. 227 - 234.

[WiCL91] Widom, J., Cochrane, R.J., Lindsay, B.G., Implementing Set-Oriented Production Rules as an Extension to Starburst, in: Proceedings of the 17th International Conference on Very Large Databases, pp. 275 - 285. 


\section{Questions \& answers}

Question []:

Why did you choose to use "else" in your rule syntax instead of "event_not_condition_action?"

Answer [H. Herbst]:

More redudancy.

Question []:

What is the point of specialization?

Answer [H. Herbst]:

Information encapsulation and reusability. 